\title{
Broadband Sharp 90-degree Bends and T-Splitters in Plasmonic Coaxial Waveguides
}

\author{
Wonseok Shin, ${ }^{\dagger}$ Wenshan Cai, ${ }^{\ddagger}$ Peter B. Catrysse, ${ }^{\dagger}$ Georgios Veronis, ${ }^{\S}$ Mark L. Brongersma, ${ }^{\|}$ \\ and Shanhui Fan*, ${ }^{\dagger}$ \\ ${ }^{\dagger}$ E. L. Ginzton Laboratory, Stanford University, Stanford, California 94305, United States \\ ${ }^{\ddagger}$ Electrical and Computer Engineering, Georgia Institute of Technology, Atlanta, Georgia 30332, United States \\ ${ }^{\S}$ Electrical and Computer Engineering, Louisiana State University, Baton Rouge, Louisiana 70803, United States \\ "Geballe Laboratory for Advanced Materials, Stanford University, Stanford, California 94305, United States
}

ABSTRACT: We demonstrate numerically that sharp $90^{\circ}$ bends and T-splitters can be designed in plasmonic coaxial waveguides at deep-subwavelength scale to operate without reflection and radiation over a broad range of wavelengths, including the telecommunication wavelength of $1.55 \mu \mathrm{m}$. We explain the principles of the operation using a transmission line model of the waveguide in the quasi-static limit. The compact bends and $\mathrm{T}$-splitters open up a new avenue for the design of densely integrated optical circuits with minimal crosstalk.
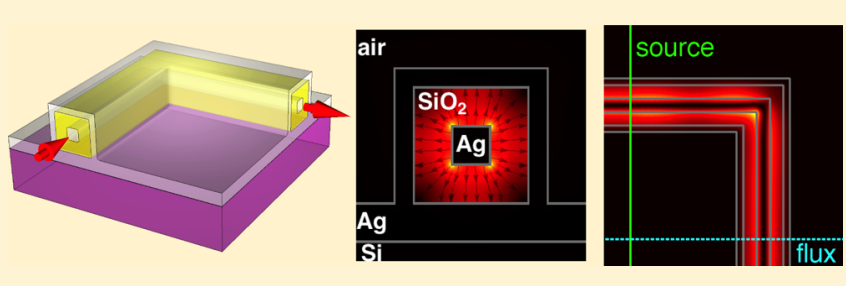

KEYWORDS: plasmonics, coaxial waveguide, transmission line, bend, splitter, integrated optical circuit

$\mathrm{R}$ outing of light in arbitrary directions inside a submicrometer-scale volume is one of the most basic functions sought after in nanophotonics. ${ }^{1-3}$ Plasmonic waveguides, despite Ohmic loss inherent in metals, have therefore been considered important components of nanophotonics due to their capability of guiding light through deep-subwavelength mode areas. ${ }^{4-9}$ A natural question arose as to whether basic waveguide components such as sharp $90^{\circ}$ bends and T-splitters can be constructed in plasmonic waveguides in a simple and compact manner. In two-dimensional (2D) metal-dielectricmetal (MDM) waveguides, it was numerically demonstrated that these components can bend and split input power almost perfectly without introducing additional reflection and radiation loss on top of the inherent Ohmic loss of the straight waveguide. $^{10}$

In realistic three-dimensional (3D) plasmonic waveguides, however, it turns out to be significantly more difficult to design sharp $90^{\circ}$ bends and T-splitters without additional loss into undesirable channels such as reflection and radiation. For example, plasmonic slot waveguides, ${ }^{11}$ which are $3 \mathrm{D}$ analogues of the 2D MDM waveguides, suffer from substantial reflection of about $16 \%$ when bent sharply by $90^{\circ}$ for near-infrared wavelengths. ${ }^{12}$ The reflection can be suppressed by rounding the sharp corner and hence increasing the bending radius of curvature slightly, ${ }^{12,13}$ but there always exists loss into radiation and surface waves. ${ }^{14}$ These extra loss channels also induce unwanted crosstalk between optical components; such crosstalk can be especially detrimental in densely integrated optical circuits, where optical components are placed close to each other. $^{15}$
$\mathrm{V}$-grooves are another type of plasmonic waveguides for which nearly perfect transmission of optical waves through sharp $90^{\circ}$ bends was reported. ${ }^{16}$ However, the lossless transmission through the bends in the $\mathrm{V}$-grooves is narrowbanded because it relies on an interference phenomenon. ${ }^{17}$ Moreover, unless the taper angles of the V-grooves are very narrow, the modes of the $\mathrm{V}$-grooves may not be at deepsubwavelength scale. ${ }^{5,18,19}$

In this Letter, we propose to use a different type of plasmonic waveguides, namely, plasmonic coaxial waveguides, for implementing sharp $90^{\circ}$ bends and T-splitters. The plasmonic coaxial waveguides have been studied both theoretically ${ }^{20,21}$ and experimentally, ${ }^{22}$ and they have been applied to achieve a variety of novel functions such as deep-subwavelength focusing, ${ }^{23,24}$ enhanced transmission, ${ }^{25-28}$ and negative refraction. ${ }^{29-31}$ In addition to this repertoire of applications, we demonstrate that the plasmonic coaxial waveguides are also useful for building sharp $90^{\circ}$ bends and T-splitters that experience nearly no loss other than the inherent Ohmic loss of the straight waveguide itself over a broad range of wavelengths, including the telecommunication wavelength of $1.55 \mu \mathrm{m}$. The structures of a bend and T-splitter are described in Figure 1a and $b$.

Unlike most of the previous works, ${ }^{20-24,26-31}$ we use coaxial waveguides with square rather than circular cross sections because they are easier to fabricate using lithography-based fabrication techniques. The cross section of the "reference

Received: June 26, 2013

Revised: August 23, 2013

Published: August 27, 2013 


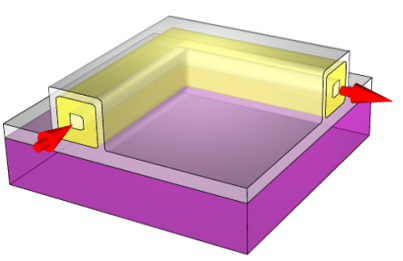

(a) Bend

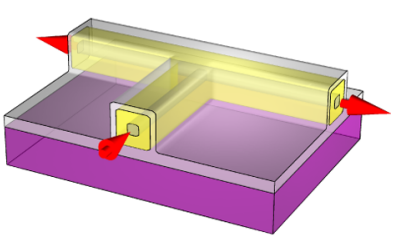

(b) Splitter

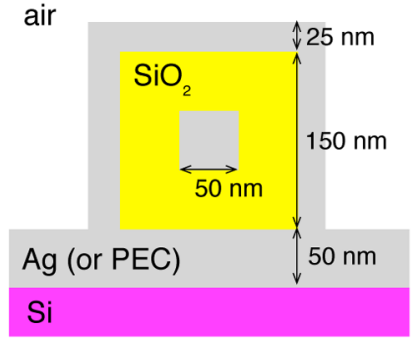

(c) Waveguide cross section

Figure 1. Structures of a sharp $90^{\circ}$ bend and T-splitter in plasmonic coaxial waveguides. ( $\mathrm{a}$ and $\mathrm{b}$ ) Structures of a bend and T-splitter with the propagation directions of light indicated by red arrows. (c) Cross section of the reference plasmonic coaxial waveguide. Silver is used as the metal, but it is substituted by perfect electric conductor (PEC) to illustrate some of the physics.

waveguide" examined in this Letter is described in Figure 1c. The waveguide is placed on top of a silicon $(\mathrm{Si})$ substrate, and the space between inner and outer coaxial metals is filled with silica $\left(\mathrm{SiO}_{2}\right)$. Throughout this Letter, we use silver $(\mathrm{Ag})$ as the metal. We also use the perfect electric conductor (PEC) to illustrate some of the physics. The choice of the metal used will be specified explicitly for each numerical result.

To study the properties of the reference waveguide as well as the bends and splitters implemented in it, we use the 3D finitedifference frequency-domain (FDFD) method to solve Maxwell's equations numerically for each vacuum wavelength $\lambda_{0}{ }^{32,33}$ The FDFD method allows us to use tabulated dielectric constants of dispersive materials such as silver ${ }^{34}$ directly, including both the real and the imaginary parts. For accurate yet efficient solution, we use nonuniform spatial grids whose cells are as small as $1 \mathrm{~nm}$ inside the waveguide region and as large as $20 \mathrm{~nm}$ further outside. To simulate infinitely long waveguides, we surround the entire simulation domain by the stretched-coordinate perfectly matched layer (SC-PML), which, for frequency-domain solvers, was proven to be much superior to the more commonly used uniaxial PML. ${ }^{35}$

The fundamental mode of the reference waveguide made of silver is a quasi-transverse-electromagnetic (quasi-TEM) mode. The quasi-TEM mode inherits many desirable properties of the true TEM mode of the same coaxial waveguide made of PEC. ${ }^{11,20}$ First, it has a nearly linear dispersion relation as shown in Figure 2a. Therefore, the waveguide has a nearly constant group velocity over a broad range of wavelengths and has no cutoff wavelength; the latter means that it can guide light with wavelengths much larger than the cross-sectional dimensions of the coaxial waveguide. Second, the $E$ - and $H$ fields of the quasi-TEM mode are tightly confined between the two metals, as shown in Figure $2 b$ and $c$ for a vacuum wavelength $\lambda_{0}=1.55 \mu \mathrm{m}$. Remarkably, we find that only a 25nm-thick outer metal layer is sufficient for confining the fields within the waveguide, because the opposite electric charges and

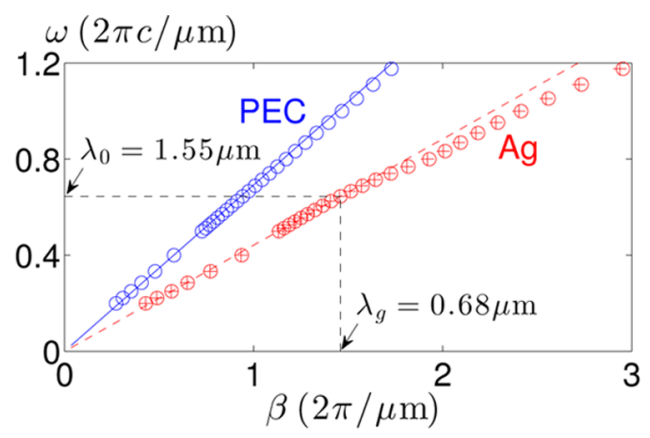

(a) Dispersion relation

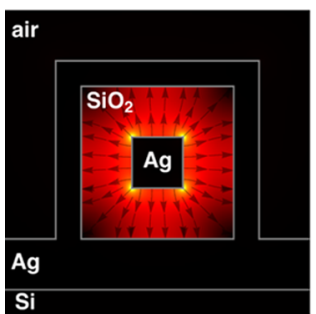

(b) Transverse $E$-field

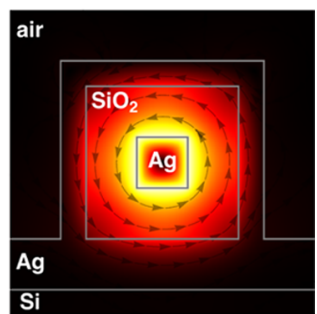

(c) Transverse $H$-field
Figure 2. Properties of the reference waveguide. (a) Dispersion relations of the fundamental modes of the reference waveguides made of silver and PEC. The data points are obtained from the FDFD mode solver. $^{11,36}$ The blue solid line is the light line in silica, and its alignment with the blue open dots proves the accuracy of the mode solver results. The red open dots and cross hairs are for the waveguides with 25-nm-thick and infinitely thick outer silver layers, respectively, and their coincidence proves the effectiveness of the 25-nm-thick outer silver layer. The red dashed line connects the origin and the data point for $\lambda_{0}=1.55 \mu \mathrm{m}$, for which the guide wavelength is $\lambda_{\mathrm{g}}=0.68 \mu \mathrm{m}$, and its alignment with the red open dots shows that the mode is quasiTEM. The propagation length ${ }^{11}$ for $\lambda_{0}=1.55 \mu \mathrm{m}$ is $L_{\mathrm{p}}=6.82 \mu \mathrm{m}$. (b and c) Magnitudes (colors) and directions (arrows) of the transverse $E$ - and $H$-fields of the fundamental mode of the reference waveguide made of silver for $\lambda_{0}=1.55 \mu \mathrm{m}$. The longitudinal components are not shown because they are much smaller than the transverse components. The dielectric constants of silicon, ${ }^{37}$ silica, ${ }^{37}$ and silver ${ }^{34}$ are taken from tabulated data.

currents carried by the inner and outer metals cancel the fields outside the coaxial waveguide efficiently. Note that the area of the silica region, where most of the fields are confined, is less than $(1.55 \mu \mathrm{m} / 10)^{2}$, which is at deep-subwavelength scale for the vacuum wavelength $\lambda_{0}=1.55 \mu \mathrm{m}$. In fact, because of the above-mentioned field cancellation effect, the area of confinement stays almost the same even if $\lambda_{0}$ increases from $1.55 \mu \mathrm{m}$, so the confinement becomes even stronger for longer wavelengths.

Now, we examine the performance of the sharp $90^{\circ}$ bend in the reference waveguide made of silver shown in Figure 1a. For each vacuum wavelength $\lambda_{0}$ between 1 and $5 \mu \mathrm{m}$, we excite the fundamental mode of the waveguide by an electric current source plane located $0.5 \mu \mathrm{m}$ before the bend; in reality the fundamental mode can be excited by coupling the lowest-order transverse magnetic $\left(\mathrm{TM}_{01}\right)$ mode of optical fibers ${ }^{38}$ or the quasi-TEM mode of plasmonic coaxial lasers. ${ }^{39}$ We then measure the transmitted power through a flux plane located 0.5 $\mu \mathrm{m}$ after the bend.

Figures $3 \mathrm{a}$ and $\mathrm{b}$ shows the solutions of Maxwell's equations for such measurement for $\lambda_{0}=1 \mu \mathrm{m}$ and $\lambda_{0}=1.55 \mu \mathrm{m}$; notice that for $\lambda_{0}=1 \mu \mathrm{m}$ a strong standing wave pattern is formed in 


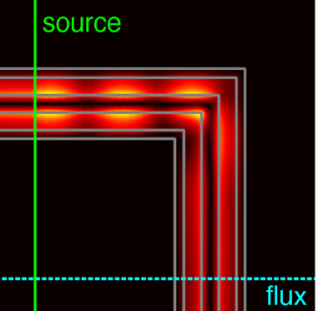

(a) $\lambda_{0}=1 \mu \mathrm{m}$

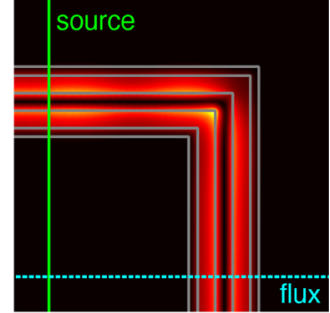

(b) $\lambda_{0}=1.55 \mu \mathrm{m}$

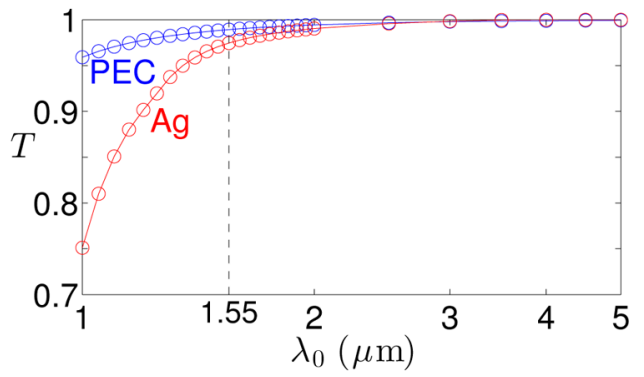

(c) Transmittance through the bend

Figure 3. Performance of the sharp $90^{\circ}$ bend. ( $a$ and b) Plots of the amplitude of the $H$-field component normal to the plane containing the axes of the input and output waveguides for $\lambda_{0}=1 \mu \mathrm{m}$ and $\lambda_{0}=$ $1.55 \mu \mathrm{m}$. Silver is used as the metal. The distances from the center of the junction to the electric current source plane (green) and the flux measurement plane (cyan) are both $0.5 \mu \mathrm{m}$. Notice the strong standing wave pattern in the input waveguide in (a) due to significant reflection of the input wave at the junction. (c) Transmittance spectra through the bend. The transmittance is higher for the PEC waveguide than the silver waveguide, but both converge to $100 \%$ as the vacuum wavelength $\lambda_{0}$ increases. For the silver waveguide, $T=97.5 \%$ is obtained at $\lambda_{0}=1.55 \mu \mathrm{m}$. The nearly perfect transmission is achieved for $\lambda_{0} \geq 1.55 \mu \mathrm{m}$, a broad range of wavelengths; note that the horizontal axis is on a logarithmic scale.

the input waveguide by the interference between the input and reflected waves, whereas for $\lambda_{0}=1.55 \mu \mathrm{m}$ the pattern is diminished significantly, which means that most of the input power is transmitted through the bend without reflection. We perform a similar measurement of the power transmitted over $(0.5+0.5) \mu \mathrm{m}$ in a straight waveguide. The ratio of the measurement in the bend with respect to the measurement in the straight waveguide is the transmittance of the bend. Such a definition of transmittance is intended to capture loss that is added on top of the propagation loss of the straight waveguide; the additional loss can be due to additional Ohmic loss introduced by the bend or due to reflection and radiation loss at the bend.

The measured transmittance is shown as a spectrum in Figure 3c. It shows that the transmittance approaches $100 \%$ as the wavelength increases. Especially, a transmittance of $97.5 \%$ is achieved for $\lambda_{0}=1.55 \mu \mathrm{m}$ without any optimization of the geometry of the bend. This remarkable phenomenon can be explained as follows. For wavelengths much larger than the cross-sectional dimensions of the waveguide, the quasi-static approximation applies, and hence the junction between the input and output waveguides at the bend can be accurately modeled as a junction between two transmission lines. ${ }^{40}$ Because the input and output waveguides have the same crosssectional shape, the two transmission lines have the same characteristic impedance. Therefore, the transmittance should be $100 \%$ in the quasi-static limit. The perfect transmission in the quasi-static limit also occurs in a bend in the reference waveguide made of PEC as shown in Figure 3c, which is consistent with the result in the classical microwave literature. ${ }^{41}$

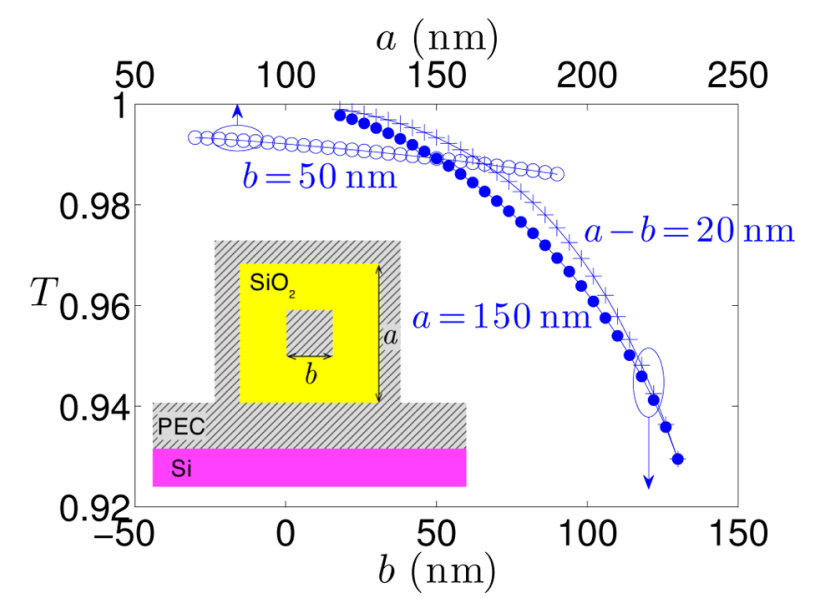

Figure 4. Performance of the sharp $90^{\circ}$ bend in the PEC coaxial waveguide with various cross-sectional dimensions. The parameters $a$ and $b$ are indicated in the inset figure. The transmittance is measured while varying $a$ for $b=50 \mathrm{~nm}$ (open dots), varying $b$ for $a=150 \mathrm{~nm}$ (solid dots), and varying $b$ for $a-b=20 \mathrm{~nm}$ (cross hairs). Note that the high transmittance for $b=50 \mathrm{~nm}$ is not very sensitive to the variation of $a$. On the other hand, the variation of $b$ affects the transmittance significantly.

We note that the nearly perfect transmission is a broadband phenomenon achieved from $\lambda_{0}=1.55 \mu \mathrm{m}$ to mid-infrared, because the quasi-static approximation applies to any sufficiently long wavelengths. The closed structure of the coaxial waveguide that prohibits coupling with other leakage channels such as radiation is crucial for the nearly perfect transmission; in other conventional 3D plasmonic waveguides that are open to the leakage channels, the transmission does not become perfect in the quasi-static limit because additional loss into the leakage channels is unavoidable.

Even though the quasi-static approximation holds in general when the cross-sectional dimensions of the coaxial waveguide are much smaller than the wavelength, we find that the size of the inner metal is the most critical among all cross-sectional dimensions. In Figure 4, we measure the transmittance through the PEC coaxial waveguide bend for a vacuum wavelength $\lambda_{0}=$ $1.55 \mu \mathrm{m}$ as varying cross-sectional dimensions of the waveguide while maintaining one of the following three parameters the same: $a, b$, and $a-b$ (see the inset of Figure 4 ). The figure shows that the transmittance stays high as long as $b$ is much smaller than the wavelength, whereas it decreases fast as $b$ increases even if the other two parameters are at deepsubwavelength scale. The strong impact of $b$, that is, the size of the inner metal, on the transmittance can be explained by the electromagnetic field distributions of the waveguide mode shown in Figure $2 \mathrm{~b}$ and $\mathrm{c}$ : the fields are strongest near the inner metal, so the size of the inner metal is likely to affect transmission properties significantly. We note, however, that the nearly perfect transmission is still quite tolerant of the changes in parameter values; for example, when $b$ changes by $10 \%$ from $50 \mathrm{~nm}$, the transmittance stays above $98 \%$ for both $a$ $=150 \mathrm{~nm}$ and $a-b=20 \mathrm{~nm}$.

Next, we examine the performance of the T-splitter in the reference waveguide made of silver shown in Figure $1 \mathrm{~b}$. By the 


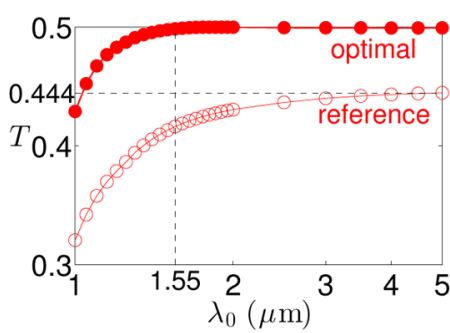

(a) Transmittance

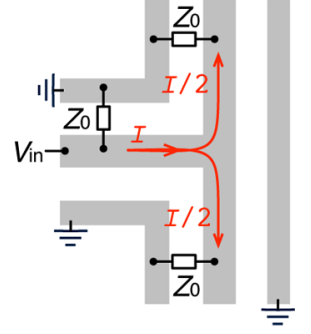

(b) Current flow
Figure 5. Performance of the T-splitter. (a) Transmittance into one of the two output waveguides of the splitter shown in Figure 1b. Silver is used as the metal. Both the output waveguides are the reference waveguides, while the input waveguide is either the reference or the optimal (see Figure 6) waveguide. For the reference input waveguide the transmittance reaches $44.4 \%$ as the vacuum wavelength $\lambda_{0}$ increases, whereas for the optimal input waveguide it reaches the ideal $50.0 \%$ over a broad range of wavelengths of $\lambda_{0} \geq 1.55 \mu \mathrm{m}$; note that the horizontal axis is on a logarithmic scale. (b) Flow of electric current in the $T$-junction, in which the three reference waveguides are modeled as three transmission lines with the same characteristic impedance $Z_{0}$. When the voltage difference $V_{\text {in }}$ is applied between the inner and outer metals, the current $I$ is launched in the input waveguide and equally divided into the two output waveguides at the junction. Note that the three seemingly separate outer metal pieces shown in the figure are actually a single connected outer metal layer.

same method used for the bend, we measure the transmittance through one of the two output waveguides. An ideal splitter should transmit $50 \%$ of the input power into each output waveguide. However, for the splitter shown in Figure $1 \mathrm{~b}$ for which the input and output waveguides have the same crosssectional shape, it turns out that the transmittance reaches only $44.4 \%$ even for long wavelengths as shown in Figure 5a. This asymptotic value of the transmittance can be also explained by modeling the $\mathrm{T}$-junction as a junction between three transmission lines in the quasi-static limit. Because all of the three waveguides connected at the $\mathrm{T}$-junction have the same cross-sectional shape, the characteristic impedance $Z_{\text {in }}$ of the input transmission line and $Z_{\text {out }}$ of each of the two output transmission lines have the same value $Z_{0}$, that is, $Z_{\text {in }}=Z_{\text {out }}=$ $Z_{0}$. The two output transmission lines form a parallel combination of impedances with respect to the input transmission line, because the current flowing through the input transmission line is equally divided into the two output transmission lines as indicated in Figure $5 \mathrm{~b}$. Therefore, the load impedance seen by the input transmission line is $Z_{L}=Z_{\text {out }} / 2$, and the reflectance is calculated as

$$
R=\frac{\left|Z_{L}-Z_{\text {in }}\right|^{2}}{\left|Z_{L}+Z_{\text {in }}\right|^{2}}=\frac{\left|Z_{0} / 2-Z_{0}\right|^{2}}{\left|Z_{0} / 2+Z_{0}\right|^{2}}=\frac{1}{9} .
$$

The remaining $1-R$ portion of the input power that is not reflected should be equally divided into each output transmission line, because no leakage channels such as radiation are coupled at the junction. Therefore, the transmittance into each output transmission line should be $T=(1-R) / 2=4 / 9=$ $44.4 \%$ in the quasi-static limit, which is exactly the asymptotic value in Figure 5a.

Equation 1 suggests one way of eliminating the reflection, which is to decrease $Z_{\text {in }}$ from $Z_{0}$ to $Z_{0} / 2$. We note that for the Tsplitter in the 2D MDM waveguide the recipe was opposite, that is, to increase $Z_{\text {in }}$, because the two output waveguides formed a series rather a parallel combination of impedances. ${ }^{10}$

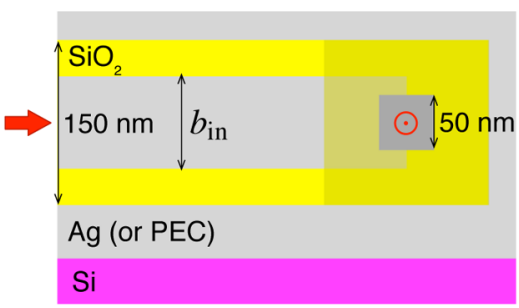

(a) Side view

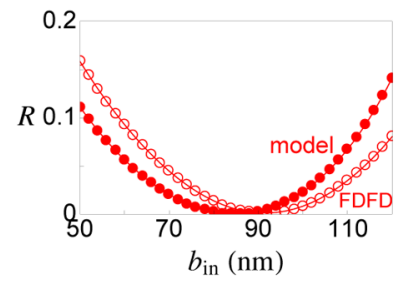

(b) $\mathrm{Ag}$

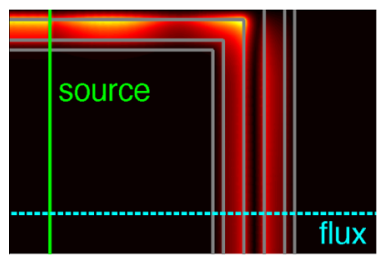

(d) $b_{\text {in }}=50 \mathrm{~nm}$

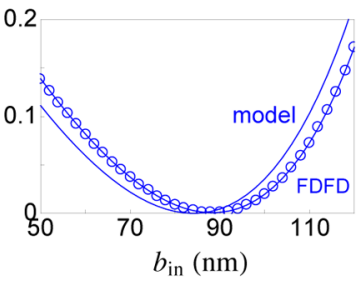

(c) PEC

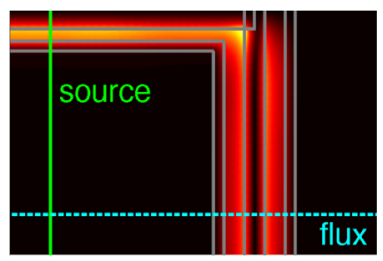

(e) $b_{\text {in }}=90 \mathrm{~nm}$

Figure 6. Optimization of the T-splitter. (a) Structure of the T-splitter on the vertical cross section containing the axis of the input waveguide. The inner metal size $b_{\text {in }}$ of the input waveguide is increased from the original size $50 \mathrm{~nm}$ to eliminate the reflection of the input wave at the $\mathrm{T}$-junction. The red arrow and bullseye indicate the propagation direction of light. (b) Spectra of the actual reflectance in the 3D FDFD solution of Maxwell's equations (open dots) and the reflectance predicted by the transmission line model (solid dots) for the T-splitter in the silver coaxial waveguide. The actual reflectance almost vanishes for $b_{\text {in }}=90 \mathrm{~nm}$, but the transmission line model slightly underestimates the optimal $b_{\text {in }}$ (c) Similar spectra for the T-splitter in the PEC coaxial waveguide, but the prediction by the transmission line model is made using the analytic formula of the characteristic impedance of the PEC coaxial waveguide. ( $\mathrm{d}$ and e) Amplitude of the $H$-field component normal to the plane containing the axes of the input and output waveguides of the splitters with the reference $\left(b_{\text {in }}=\right.$ $50 \mathrm{~nm})$ and optimal $\left(b_{\mathrm{in}}=90 \mathrm{~nm}\right)$ input waveguides. Only a half of the splitter is shown by virtue of the mirror symmetry. Silver is used as the metal, and the distances from the center of the junction to the electric current source plane (green) and the flux measurement plane (cyan) are both $0.5 \mu \mathrm{m}$. Notice the strong standing wave pattern in (d) due to significant reflection of the input wave at the $\mathrm{T}$-junction.

It is important to emphasize that the plasmonic coaxial waveguides and 2D MDM waveguides have very different topologies in their transmission line models for the T-splitters, despite a close connection between these two classes of waveguides in terms of the modal properties of the straight waveguides. $^{21}$

To eliminate the reflection, we decrease $Z_{\text {in }}$ gradually by increasing the size $b_{\text {in }}$ of the inner metal of the input waveguide as described in Figure 6a and measure the reflectance for $\lambda_{0}=$ $1.55 \mu \mathrm{m}$. Figure $6 \mathrm{~b}$ shows that indeed the reflection almost vanishes for $b_{\text {in }}=90 \mathrm{~nm}$, for which the reflectance is only $0.17 \%$. We note that the nearly vanishing reflection is quite tolerant of the change in $b_{\text {in }}$; for example, even if $b_{\text {in }}$ changes by $10 \%$ from the optimal value, the reflectance stays below $2 \%$. 
Figure $6 \mathrm{~d}$ and e shows the solutions of Maxwell's equations for $b_{\text {in }}=50 \mathrm{~nm}$ and $b_{\text {in }}=90 \mathrm{~nm}$ for $\lambda_{0}=1.55 \mu \mathrm{m}$. Notice the presence of a strong standing wave pattern in the input waveguide in Figure $6 \mathrm{~d}$ and the absence of such a standing wave pattern in Figure $6 \mathrm{e}$, which confirms that the choice of $b_{\text {in }}$ $=90 \mathrm{~nm}$ indeed results in nearly perfect transmission.

Even though the splitter is optimized for $\lambda_{0}=1.55 \mu \mathrm{m}$, the same splitter turns out to exhibit nearly perfect transmission over a broad range of wavelengths spanning from $\lambda_{0}=1.55 \mu \mathrm{m}$ to mid-infrared as shown in Figure 5a. The broadband perfect transmission is also explained by the transmission line model as follows. Because the mode of each plasmonic coaxial waveguide is quasi-TEM, its characteristic impedance is nearly independent of wavelength. Therefore, for sufficiently long wavelengths for which the transmission line model holds, eq 1 predicts the nearly constant transmittance, which is $T=50 \%$ for the optimized splitter.

To assess the validity of the transmission line model more quantitatively, we predict the reflectance by substituting numerically calculated impedances $Z$ in the formula for $R$ in eq $1 ; Z$ is calculated as $V / I$, where the voltage difference $V$ between the inner and the outer metals and the electric current I flowing through the inner metal are calculated by numerically integrating the $E$ - and $H$-fields of the fundamental mode of the waveguide. $^{12,42}$ In Figure $6 \mathrm{~b}$, the predicted reflectance is compared with the actual reflectance in the 3D FDFD solution of Maxwell's equations. In Figure 6c we make a similar comparison between the predicted and actual reflectances for the splitter in the PEC coaxial waveguide, for which the analytic expression for $Z$ exists. ${ }^{43-46}$ For both the silver and the PEC coaxial waveguides, the prediction from the transmission line model correctly describes the overall trend of the actual reflectance as a function of $b_{\text {in }}$, but we find that the model slightly underestimates the optimal $b_{\text {in }}$ for vanishing reflectance.

In addition to its dependence on the impedances of the input and output waveguides, the reflectance also depends on the detailed shape of the junction between the input and output waveguides: it turns out that the reflectance does not reduce to zero unless the thicker inner metal of the input waveguide extends into the junction and reaches the central axis of the output waveguide as shown in Figure 6a. Such dependence on the detailed shape of the junction is of course not incorporated in the simple transmission line model above. Following the literature of microwave circuits one could develop a more sophisticated model that includes additional lumped circuit elements $^{47,48}$ to describe the properties of the junction more accurately. Nevertheless, as we can see here the simple model above does provide very important guidance for the overall design of the T-splitter.

In conclusion, we have proposed and analyzed compact and realistic designs of sharp $90^{\circ}$ bends and T-splitters in plasmonic coaxial waveguides, which can be useful in delivering optical signals into a submicrometer-scale volume such as a nanophotonic detector. The bends and splitters transmit optical waves nearly perfectly without parasitic reflection and radiation, so they help to minimize undesirable crosstalk in integrated optical circuits. Also, because the nearly perfect transmission occurs over a broad range of wavelengths, the performance of our bends and splitters should be tolerant of fabrication errors, thermal expansion, and wavelength detuning than previously proposed bend and splitter designs based on interference and resonance. The proposed designs can be fabricated either by standard lithography-based fabrication methods, or by more sophisticated methods utilizing silver nanowire bends ${ }^{49,50}$ and dielectric-coated silver nanowires. ${ }^{51-53}$ Another interesting approach is to use metal-coated semiconductor nanowires ${ }^{54,55}$ as conductive inner pieces of plasmonic coaxial waveguides; because the semiconductor nanowires can be grown into various structures such as bends, ${ }^{56}$ branches, ${ }^{57,58}$ and combs, ${ }^{59}$ they can be useful for building networks of plasmonic coaxial waveguides. We believe that our theoretical study will inspire further experimental works on actual fabrication and characterization of complex optical circuits based on plasmonic waveguides.

\section{AUTHOR INFORMATION}

\section{Corresponding Author}

*E-mail: shanhui@stanford.edu.

\section{Notes}

The authors declare no competing financial interest.

\section{ACKNOWLEDGMENTS}

This work was supported by the National Science Foundation (Grant No. DMS 09-68809), the MARCO Interconnect Focus Center, and the AFOSR MURI programs (Grant Nos. FA955009-1-0704 and FA9550-12-1-0024). W.S. also acknowledges the support of Samsung Scholarship.

\section{REFERENCES}

(1) Joannopoulos, J. D.; Johnson, S. G.; Winn, J. N.; Meade, R. D. Photonic Crystals: Molding the Flow of Light, 2nd ed.; Princeton University Press: Princeton, NJ, 2008.

(2) Manolatou, C.; Johnson, S. G.; Fan, S.; Villeneuve, P. R.; Haus, H. A.; Joannopoulos, J. D. J. Lightwave Technol. 1999, 17, 1682-1692.

(3) Miller, D. A. B. Proc. IEEE 2000, 88, 728-749.

(4) Barnes, W. L.; Dereux, A.; Ebbesen, T. W. Nature 2003, 424, 824-830.

(5) Ozbay, E. Science 2006, 311, 189-193.

(6) Maier, S. IEEE J. Sel. Top. Quantum Electron. 2006, 12, 16711677.

(7) Oulton, R. F.; Sorger, V.; Genov, D.; Pile, D. F. P.; Zhang, X. Nat. Photonics 2008, 2, 496-500.

(8) Brongersma, M. L.; Shalaev, V. M. Science 2010, 328, 440-441.

(9) Gramotnev, D. K.; Bozhevolnyi, S. I. Nat. Photonics 2010, 4, 8391.

(10) Veronis, G.; Fan, S. Appl. Phys. Lett. 2005, 87, 131102

(11) Veronis, G.; Fan, S. J. Lightwave Technol. 2007, 25, 2511-2521.

(12) Cai, W.; Shin, W.; Fan, S.; Brongersma, M. L. Adv. Mater. 2010, $22,5120-5124$

(13) Liu, L.; Han, Z.; He, S. Opt. Express 2005, 13, 6645-6650.

(14) Shin, W.; Raman, A.; Fan, S. J. Opt. Soc. Am., B 2012, 29, 1048 1054.

(15) Veronis, G.; Fan, S. Opt. Express 2008, 16, 2129-2140.

(16) Pile, D. F. P.; Gramotnev, D. K. Opt. Lett. 2005, 30, 1186-1188.

(17) Volkov, V. S.; Bozhevolnyi, S. I.; Devaux, E.; Ebbesen, T. W. Appl. Phys. Lett. 2006, 89, 143108.

(18) Pile, D. F. P.; Gramotnev, D. K. Opt. Lett. 2004, 29, 1069-1071.

(19) Moreno, E.; Garcia-Vidal, F. J.; Rodrigo, S. G.; Martin-Moreno, L.; Bozhevolnyi, S. I. Opt. Lett. 2006, 31, 3447-3449.

(20) Baida, F. I.; Belkhir, A.; Van Labeke, D.; Lamrous, O. Phys. Rev. B 2006, 74, 205419.

(21) Catrysse, P. B.; Fan, S. Appl. Phys. Lett. 2009, 94, 231111.

(22) de Waele, R.; Burgos, S. P.; Polman, A.; Atwater, H. A. Nano Lett. 2009, 9, 2832-2837.

(23) Rusina, A.; Durach, M.; Nelson, K. A.; Stockman, M. I. Opt. Express 2008, 16, 18576-18589.

(24) Weber-Bargioni, A.; Schwartzberg, A.; Cornaglia, M.; Ismach, A.; Urban, J. J.; Pang, Y.; Gordon, R.; Bokor, J.; Salmeron, M. B.; 
Ogletree, D. F.; Ashby, P.; Cabrini, S.; Schuck, P. J. Nano Lett. 2011, 11, 1201-1207.

(25) Moreau, A.; Granet, G.; Baida, F. I.; Van Labeke, D. Opt. Express 2003, 11, 1131-1136.

(26) Baida, F. I.; Van Labeke, D.; Granet, G.; Moreau, A.; Belkhir, A. Appl. Phys., B: Lasers Opt. 2004, 79, 1-8.

(27) Baida, F. I. Appl. Phys., B: Lasers Opt. 2007, 89, 145-149.

(28) Rybczynski, J.; Kempa, K.; Herczynski, A.; Wang, Y.; Naughton, M. J.; Ren, Z. F.; Huang, Z. P.; Cai, D.; Giersig, M. Appl. Phys. Lett. 2007, 90, 021104.

(29) Rodríguez-Fortuño, F. J.; García-Meca, C.; Ortuño, R.; Martí, J.; Martínez, A. Opt. Lett. 2009, 34, 3325-3327.

(30) Burgos, S. P.; de Waele, R.; Polman, A.; Atwater, H. A. Nat. Mater. 2010, 9, 407-412.

(31) de Waele, R.; Burgos, S. P.; Atwater, H. A.; Polman, A. Opt. Express 2010, 18, 12770-12778.

(32) Veronis, G.; Fan, S. In Surface Plasmon Nanophotonics; Brongersma, M., Kik, P., Eds.; Springer: Dordrecht, Netherlands, 2007; pp 169-182.

(33) Shin, W.; Fan, S. Opt. Express 2013, 21, 22578-22595.

(34) Lide, D. R., Ed. CRC Handbook of Chemistry and Physics, 88th ed.; CRC Press: Boca Raton, FL, 2007.

(35) Shin, W.; Fan, S. J. Comput. Phys. 2012, 231, 3406-3431.

(36) Pereda, J. A.; Vegas, A.; Prieto, A. Microwave Opt. Technol. Lett. 2003, 38, 331-335.

(37) Palik, E. D., Ed. Handbook of Optical Constants of Solids; Academic Press: San Diego, CA, 1985.

(38) Ramachandran, S.; Kristensen, P.; Yan, M. F. Opt. Lett. 2009, 34, 2525-2527.

(39) Khajavikhan, M.; Simic, A.; Katz, M.; Lee, J. H.; Slutsky, B.; Mizrahi, A.; Lomakin, V.; Fainman, Y. Nature 2012, 482, 204-207.

(40) Marcuvitz, N. Waveguide Handbook; The Institution of Engineering and Technology: London, U.K., 2009.

(41) Navarro, E. A.; Wu, C.; Chung, P. Y.; Litva, J. Microwave Opt. Technol. Lett. 1995, 8, 138-140.

(42) Huang, J. S.; Feichtner, T.; Biagioni, P.; Hecht, B. Nano Lett. 2009, 9, 1897-1902.

(43) Conning, S. W. IEEE Trans. Microwave Theory Tech. 1964, 12, $468-468$.

(44) Anderson, G. M. Trans. Am. Inst. Elect. Eng. 1950, 69, 728-731.

(45) Bowman, F. Introduction to Elliptic Functions with Applications; Dover: New York, 1961.

(46) Wadel, B. C. Transmission Line Design Handbook; Artech House: Norwood, MA, 1991; Sec. 3.2.4.

(47) Montgomery, C. G., Dicke, R. H., Purcell, E. M., Eds. Principles of Microwave Circuits; McGraw-Hill: New York, 1948; Sec. 9.6.

(48) Garg, R. In Encyclopedia of RF and Microwave Engineering; Chang, K., Ed.; John Wiley \& Sons: Hoboken, NJ, 2005; pp 653-658.

(49) Sanders, A. W.; Routenberg, D. A.; Wiley, B. J.; Xia, Y.; Dufresne, E. R.; Reed, M. A. Nano Lett. 2006, 6, 1822-1826.

(50) Wang, W.; Yang, Q.; Fan, F.; Xu, H.; Wang, Z. L. Nano Lett. 2011, 11, 1603-1608.

(51) Yin, Y.; Lu, Y.; Sun, Y.; Xia, Y. Nano Lett. 2002, 2, 427-430.

(52) Sun, X.; Li, Y. Adv. Mater. 2005, 17, 2626-2630.

(53) Luo, L.-B.; Yu, S.-H.; Qian, H.-S.; Zhou, T. J. Am. Chem. Soc. 2005, 127, 2822-2823.

(54) Fang, Y. J.; Sha, S.; Wang, Z. L.; Wan, Y. T.; Xia, W. W.; Wang, Y. W. Appl. Phys. Lett. 2011, 98, 033103.

(55) Pyne, S.; Sahoo, G. P.; Bhui, D. K.; Bar, H.; Sarkar, P.; Samanta, S.; Maity, A.; Misra, A. Spectrochim. Acta, Part A: Mol. Biomol. Spectrosc. 2012, 93, 100-105.

(56) Barrelet, C. J.; Greytak, A. B.; Lieber, C. M. Nano Lett. 2004, 4, 1981-1985.

(57) Wang, D.; Qian, F.; Yang, C.; Zhong, Z.; Lieber, C. M. Nano Lett. 2004, 4, 871-874.

(58) Dick, K. A.; Deppert, K.; Larsson, M. W.; Mårtensson, T.; Seifert, W.; Wallenberg, L. R.; Samuelson, L. Nat. Mater. 2004, 3, $380-384$.
(59) Yao, J.; Yan, H.; Lieber, C. M. Nat. Nanotechnol. 2013, 8, 329335.

\section{NOTE ADDED AFTER ASAP PUBLICATION}

Reference 33 was updated in the version published ASAP on September 4, 2013. The revised version was re-posted on September 19, 2013. 